\title{
Incentivos, motivaciones y beneficios de la incorporación de la gestión ambiental en las empresas
}

Incentives, Motivations and Benefits of Including Environmental Management in Companies

Incentivos, motivações e benefícios da incorporação da gestão ambiental nas empresas

\author{
Ingrid Yadibel Cuevas Zúniga* \\ Luis Rocha Lona ${ }^{\star *}$ \\ María del Rocío Soto Flores ${ }^{* \star \star}$
}

Fecha de recibido: 17 de marzo de 2015

Fecha de aprobado: 21 de septiembre de 2015

Doi: dx.doi.org/10.12804/rev.univ.empresa.30.2016.06

Para citar este artículo: Cuevas Zúñiga, I. Y., Rocha Lona, L. \& Soto Flores, M. R. (2016). Incentivos, motivaciones y beneficios de la incorporación de la gestión ambiental en las empresas. Universidad \& Empresa, 18(30), 121-141. Doi: dx.doi.org/10.12804/rev.univ.empresa.30.2016.06

* Maestra en Ciencias en Administración de Negocios y Contadora Pública, del Instituto Politécnico Nacional, Escuela Superior de Comercio y Administración (ESCA), Unidad Santo Tomás (México) y doctoranda en Ciencias Sociales, del Instituto Politécnico Nacional (IPN), Escuela Superior de Comercio y Administración (ESCA), Unidad Santo Tomás (México). Profesora de la Maestría en Ciencias en Administración de Negocios. Correo electrónico: icuevasz@ipn.mx

** PhD Operations Management, Manchester Business School, University of Manchester. MSc in Control Systems, University of Sheffield. Jefe de la Sección de Estudios de Posgrado e Investigación y profesor investigador del Instituto Politécnico Nacional (IPN), Escuela Superior de Comercio y Administración (ESCA), Unidad Santo Tomás (México). Correo electrónico: 1rocha@ipn.mx

*** Doctora en Administración y Dirección de Empresas, de la Universidad Politécnica de Cataluña (España). Profesora de la Sección de Estudios de Posgrado e Investigación (SEPI) de la Escuela Superior de Comercio y Administración Santo Tomás (ESCA ST) del Instituto Politécnico Nacional (IPN) (México). Correo electrónico: mrsoto03@yahoo.com.mx 


\section{RESUMEN}

La mayoría de las empresas desconoce los incentivos, las motivaciones y los beneficios de incorporar la gestión ambiental como herramienta de regulación de sus acciones de sustentabilidad. Para esta investigación, que procura responder a esa necesidad, se formuló un cuestionario a diez empresas que participan en el Programa Nacional de Auditoría Ambiental (PNAA), en México. Se encontró que los incentivos para incluirla son el corporativismo verde, los grupos de interés, el consumidor y el mercado verde. Las motivaciones, por su parte, son el perfil empresarial, los recursos y las capacidades, la estrategia de negocio y la gestión de acciones de sustentabilidad. Los beneficios son económicos, sociales, ambientales y en materia de competitividad.

Palabras clave: beneficios, gestión ambiental, incentivos, motivaciones, sustentabilidad empresarial.

\section{ABSTRACT}

The majority of companies are unaware of the incentives, motivations and benefits of including environmental management as a tool with which to regulate their sustainability actions. To carry out this research, which endeavors to satisfy this need, ten companies which participate in the National Environmental Audit Program in Mexico (NEAP) completed a questionnaire. It was found that the incentives they have to include this type of management are green corporatism, stakeholders, the consumer and the green market. The motivations, for their part, are the entrepreneurial profile, the resources and capabilities, the business strategy, and the management of sustainability actions. Finally, the benefits are economic, social, environmental and competitive.

Keywords: Benefits, environmental management, incentives, motivations, entrepreneurial sustainability.

\section{RESUMO}

A maioria das empresas desconhecem os incentivos, motivações e benefícios de incorporar a gestão ambiental como ferramenta de regulação de suas ações de sustentabilidade. Para a realização desta pesquisa, que procura responder a essa necessidade, se realizou um questionário a dez empresas que participam no Programa Nacional de Auditoria Ambiental (PNAA), no México. Se encontrou que os incentivos que têm para incorporar dita gestão são o corporativismo verde, os grupos de interesse, o consumidor e o mercado verde, as motivações, por sua parte, são o perfil empresarial, os recursos e capacidades, a estratégia de negócio e a gestão de ações de sustentabilidade. Finalmente, os benefícios são económicos, sociais, ambientais e em matéria de competitividade.

Palavras-chave: benefícios, gestão ambiental, incentivos, motivações, sustentabilidade empresarial. 


\section{INTRODUCCIÓN}

La gestión ambiental es una herramienta que permite implementar actividades encaminadas a preservar el ambiente y la calidad de vida de la sociedad, por medio de políticas e instrumentos que procuran la incorporación efectiva de una sustentabilidad corporativa. La sustentabilidad se ha tratado de vincular a las estrategias empresariales, con evidencias de que puede tener un éxito operacional moderado, puesto que las empresas reconocen su responsabilidad ante la sociedad y el ambiente y algunas de ellas han comprendido además la importancia profunda que tiene la gestión ambiental.

Estos hechos llevan a considerar la relevancia de estudiar los incentivos, las motivaciones y los beneficios de la gestión ambiental, así como su relación con la implementación de buenas prácticas de sustentabilidad por parte de las empresas (Cleveland, Stern \& Costanza, 2001).

La literatura muestra que existe una conciencia creciente en las empresas de la necesidad de asumir un compromiso con el desarrollo sustentable y que las proyecciones apuntan a que esto se fortalecerá (Lawler, Worley \& Creelman, 2011). Sin embargo, en materia sustentable, hay limitaciones que dificultan la ejecución de acciones y estrategias empresariales encaminadas a la preservación del ambiente y a mejorar la calidad de vida de la sociedad. Esto sin perder rentabilidad, restricciones como el rezago en la normatividad, la existencia de estructuras administrativas inadecuadas, una infraestructura tecnológica obsoleta, la deficiencia en materia de formación sustentable, la presencia de poca o nula inversión en materia de lo sustentable, entre otras. El reto para múltiples organizaciones es alcanzar un desarrollo en esta materia, en donde se equilibren sus objetivos económicos y ambientales y se satisfagan las necesidades sociales (Pacheco, Pavón \& Mejía, 2002).

Se ha observado que muchas empresas en México, en particular las medianas, desconocen los incentivos, las motivaciones y los beneficios de implementar acciones sustentables a partir de la gestión ambiental, concebida como una herramienta adecuada para incluir la sustentabilidad en la estrategia del negocio.

Como resultado, dichas compañías no consiguen una certificación reputada, la cual podría permitirles, entre otras cosas, incrementar su competitividad o entrar a nuevos mercados. Por esta razón, el objetivo general de esta investigación fue identificar los 
incentivos, las motivaciones y los beneficios clave para las empresas que incluyen acciones de sustentabilidad en su estrategia de negocio.

\section{REVISIÓN DE LITERATURA:} LA GESTIÓN AMBIENTAL Y LA IMPLEMENTACIÓN DE BUENAS PRÁCTICAS DE SUSTENTABILIDAD

El desarrollo sustentable ha propiciado una visión innovadora que contempla la relación entre el ser humano y su entorno mediante la preservación de este y su equilibrio con los factores sociales y económicos (Ramírez, Sánchez \& García, 2004; Sachs, 2015). Dicho concepto surgió cuando la Organización de las Naciones Unidas (ONU) creó la Comisión mundial sobre el medio ambiente y desarrollo que, en 1987, divulgó el informe Nuestro futuro común. En él, este es definido como "satisfacer las necesidades de las generaciones presentes sin comprometer las posibilidades de las del futuro para atender sus propias necesidades" (World Commission on Environment and Development [WCED], 1987).

Se han fomentado políticas y programas nacionales e internacionales de sustentabilidad (Elliott, 2006; Rogers, Jalal \& Boyd, 2008; Soubbotina, 2004; Williams, 2014a). De la mano de estas políticas, las organi- zaciones también han mostrado una preocupación creciente por los impactos que generan sus operaciones sobre el ambiente (Jacobsen, 2011; Rainey, 2006).

En buena parte del mundo, los Gobiernos y las instituciones multilaterales han implementado diversos mecanismos vinculados con estos asuntos, entre los que se destacan las certificaciones (Auld, 2014; Edwards, 2001; Gulbrandsen, 2010). Estas constituyen una medida para mejorar el desempeño ambiental de las compañías. Las organizaciones que las adquieren pueden tener mayor acceso a múltiples mercados internacionales, así como mejores oportunidades de crecimiento y desarrollo dentro de los mismos. Entre las certificaciones existentes se destacan la ISO 14000, el Eco-Management and Audit Scheme (EMAS) y la Industria limpia y Calidad ambiental, entre otras (Wayne, 2000).

Preocupado por el ambiente, el estado de México implementó el Programa Nacional de Auditoría Ambiental (PNAA), por medio de la Secretaría del Medio Ambiente y Recursos Naturales (Semarnat) y la Procuraduría Federal de Protección al Ambiente (Profepa). Este consiste en una serie ordenada de actividades tendientes a fomentar la realización de auditorías ambientales. El ingreso 
al programa es de carácter voluntario, con la finalidad no solo de garantizar el cumplimiento efectivo de la legislación, sino de incrementar la eficiencia de los procesos productivos, el desempeño ambiental y la competitividad y obtener una certificación de Industria limpia, Calidad ambiental turística o Calidad ambiental (Semarnat, 2014).

Para emitir la certificación, mediante una auditoría ambiental, la Profepa verifica aspectos como el aire, el agua, las emisiones a la atmósfera, el suelo y el subsuelo, los residuos peligrosos, los residuos sólidos e industriales no peligrosos, el ruido, la seguridad e higiene industrial, la energía, las instalaciones civiles y eléctricas, el aprovechamiento de los recursos naturales, el riesgo ambiental y los sistemas de gestión ambiental. Asimismo, establece tres enfoques para ampliar el alcance del programa:

- Enfoque integral: destaca el concepto de cadena de producción. Se pretende que las empresas certificadas fomenten la incorporación al PNAA de sus proveedores, distribuidores y clientes.

- Enfoque sectorial: incluir a los sectores industriales como la industria cementera, automotriz y cervecera en el PNAA.

- Enfoque geográfico: busca la inserción de las industrias agru- padas en parques o corredores industriales (Profepa, 2014).

Contar con esta certificación ofrece beneficios para las empresas que han visto la sustentabilidad como una oportunidad de negocio. La Profepa destaca que la compañía certificada demuestra que está cumpliendo con la legislación ambiental, lo cual le facilita los procesos de exportación, al cumplir con las leyes mexicanas en esta materia. Esto, en últimas, le otorga una mayor credibilidad. De igual forma, la empresa puede usar su certificado para hacer publicidad y, en el caso de corporaciones transnacionales, comprobar ante sus accionistas que se está cumpliendo con la normatividad del país en el que han depositado su confianza para establecer alguna filial.

En este contexto surge el "corporativismo verde", que involucra a aquellas organizaciones que cuentan con una gestión ambiental orientada a implementar medidas que preserven el ambiente (Gudynas, 1992). Las empresas compiten en un entorno global caracterizado por su incertidumbre, dinamismo y complejidad (Binder, Jänicke \& Petschow, 2001). La estrategia empresarial suele ser la encargada de transformar riesgos en oportunidades, para adaptarse al entorno e incidir positivamente en él (Chamorro, 2001). 
En la actualidad, es necesario que los empresarios, empleados y la sociedad reflexionen acerca de su responsabilidad en el cumplimiento de la premisa del desarrollo sustentable (Williams, 2014b). Se estima conveniente la generación de vínculos virtuosos en esta materia por parte de las actividades productivas, así como la toma de conciencia, en general, para poder poner en práctica una serie de alternativas tecnológicas que permitan preservar el entorno (Enders \& Remig, 2015).

Frente a los procesos de globalización, la situación se torna compleja para las organizaciones (Wild \& Wild, 2014). La sobrevivencia de la empresa depende de las decisiones que tomen los directivos de acuerdo con las condiciones que establece la economía en el entorno en donde compite (Wheelen, Hunger, Hoffman \& Bamford, 2014). Así, al incorporar los recursos naturales en el sistema económico (Daly, 1996) se define un marco que regula el nexo entre la empresa, la sociedad y el Gobierno en torno al cuidado y a la protección del ambiente. En ese momento surge el concepto de empresa sustentable (Barba \& Solís, 2000). Este tipo de compañía está basado en la construcción de una visión que procura ir más allá del rendimiento financiero y tomar en cuenta aspectos como el bienestar de la comuni- dad y la conservación ecológica, entre otros (Menon \& Menon, 1997).

La incorporación de la sustentabilidad brinda a las organizaciones una visión particular de cómo hacer su negocio de una forma diferente. Una herramienta que utilizan estas empresas es la gestión ambiental, la cual les permite un comportamiento sustentable de acuerdo con las metas fijadas y como respuesta a normas ambientales y presiones sociales, financieras y económicas (Barrow, 2006).

La gestión ambiental consiste en un proceso continuo de acciones que comienzan con la definición de la política sustentable, la planificación, la acción, la revisión y la mejora de la actuación de la empresa (Ayuntamiento de Socuéllamos, 2000).

Ortega y Rodríguez (1994) definen la gestión ambiental como el conjunto de disposiciones orientadas a mantener un capital ambiental suficiente para que la calidad de vida de las personas y el patrimonio natural sean lo más elevado posible. Este tipo de administración ayuda a la empresa a tomar conciencia de los aspectos en torno a la sustentabilidad e identificar procesos que deben ser mejorados para implantar medidas más eficaces y rentables. Además, al insertarse en la organi- 
zación, esta formará parte de su filosofía y los procedimientos tanto de la Dirección como de los demás trabajadores (Belcham, 2015).

\section{METODOLOGÍA}

Los estudios acerca de la gestión ambiental ponen de manifiesto la importancia de considerar la sustentabilidad en la estrategia de las organizaciones, con el fin de minimizar el impacto ambiental, abarcar nuevos mercados, producir bienes y servicios amigables con el ambiente y dar respuesta a la presión que ejercen los grupos de interés.

La tendencia es alcanzar un desarrollo en esta materia, en el cual las empresas busquen objetivos económicos y ambientales, a la vez que satisfacen las necesidades sociales (Barnerjee, 2002; Pacheco et al., 2002; Rushton, 2002). A partir de esta postura, Rodríguez, Villareal y Giachetti (2002) señalan la importancia de la incorporación de herramientas como la gestión ambiental, que permita a las empresas considerar la sustentabilidad en la estrategia de negocio. Pacheco, Pavón y Mejía (2002) identifican que las empresas buscan conciliar sus objetivos sociales, económicos y ambientales para impulsar buenas prácticas de sustentabilidad; no obstante, desconocen la forma de hacerlo. En sus estadísticas, la Semarnat (2014) muestra la necesidad de fomentar en las empresas la inclusión de la variable ambiental en su estrategia ambiental, pues se observó que la mayoría de las empresas en México, en particular las medianas, desconocen los incentivos, las motivaciones y los beneficios de implementar una gestión ambiental como herramienta para incorporar la sustentabilidad en la estrategia de los negocios. En consecuencia, no logran obtener una certificación ambiental que les permita incrementar su competitividad o insertarse en nuevos mercados.

Esta investigación es de naturaleza mixta (cualitativa y cuantitativa) (Teddlie \& Tashakkori, 2009), pues su finalidad fue identificar incentivos, motivaciones y beneficios que tienen las empresas para implementar una gestión ambiental en el desarrollo de sus prácticas empresariales de sustentabilidad corporativa. El trabajo se realizó desde un enfoque cualitativo, debido a que su objetivo fue la descripción de las cualidades de un fenómeno (Ritchie, 2013) e hizo uso de un cuestionario para recabar y analizar valiosa información primaria (Black, 2009).

La investigación cualitativa invita a que, además de describir y medir variables sociales, se consideren también los significados subjetivos 
y el contexto en donde ocurre el fenómeno estudiado (Hernández, Fernández \& Baptista, 2009). Los informantes básicos fueron los actores responsables de tomar decisiones acerca de la implementación de la gestión ambiental. Esta fue estimada como una fuente esencial de información.

Además, esta es una investigación de tipo documental, pues se recurrió a artículos, informes, reportes de investigación, tesis y libros relativos a la gestión ambiental, al perfil estratégico y a la sustentabilidad corporativa. Estos fueron empleados para la elaboración del marco teórico y conceptual. Asimismo, el trabajo se fundamentó en la descripción de variables sociales con base en el análisis del contexto de dicha gestión. Por último, el estudio tiene un alcance descriptivo, porque especifica las propiedades, las características y los perfiles de las empresas que participan en el PNAA.

Los datos se recolectaron con el propósito de describir variables y analizar su incidencia e interrelación en un momento dado y fueron recabados durante el mes de diciembre de 2012. En cuanto al horizonte espacial, se recurrió al estado de México y al distrito federal, por la cercanía y el número de empresas participantes en el programa.
A partid de lo anterior, se procedió a encuestar a las empresas que participan en el PNAA en el estado de México y el distrito federal, en particular, aquellas que cuentan con la certificación de Industria limpia. Se decidió que la muestra sería de 234 medianas y grandes empresas que conforman el programa, representadas por los líderes de proyectos de sustentabilidad. El sistema de recolección de información fue la base de datos propia de la Semarnat y la Profepa, recopilada hasta diciembre de 2012 y publicada en su página de Internet.

El principal problema que planteó la base de datos completa es que solo 86 empresas contaban con información de correo electrónico y solo 168 con al menos un número telefónico, de modo que no se encontró una manera efectiva de contactarlas. Los cuestionarios se enviaron por correo electrónico. De estos, se recibieron diez respuestas, lo que representó un $5,8 \%$ de la población. Además, se hicieron entrevistas semiestructuradas a dos líderes de proyectos de sustentabilidad y directores. Con ellos se discutió la forma en la que se implementa este tipo de proyectos en sus respectivas organizaciones, con la finalidad de identificar los incentivos, las motivaciones y los beneficios con los que cuentan las compañías para implementar una gestión ambiental. 


\section{RESULTADOS Y DISCUSIÓN}

Para el análisis de la información recabada se utilizó la estadística descriptiva. Esta presenta y caracteriza un conjunto de datos con el fin de describir sus atributos. La información de tipo cualitativo fue examinada con el fin de explicar, complementar y profundizar lo identificado en el cuestionario aplicado. Las categorías analizadas fueron los incentivos, las motivaciones y los beneficios de implementar una gestión ambiental.

\subsection{Incentivos}

Las razones de las organizaciones para diseñar, implementar y gestionar políticas en materia sustentable tienen que ver con el interés de cumplir con la normatividad ambiental. Esto fue indicado por la totalidad de los informantes. En efecto, $100 \%$ de las empresas las incorpora con este fin, para estar a tono con las normas y regulaciones establecidas por el Gobierno, los mercados y los múltiples programas nacionales e internacionales existentes, a pesar de que aún son poco aplicables en México y no se generan incentivos fiscales para las empresas. Otra razón por la que las empresas implementan este tipo de gestión, válida en un $70 \%$ de los casos, es ofrecer un producto o servicio amigable con el ambiente.
Un $60 \%$ de las empresas encuestadas sigue un lineamiento estratégico establecido por el corporativo, el cual exige el cumplimiento de las políticas en favor de la sustentabilidad. Un 10\% implementa este tipo de administración ambiental debido a los beneficios económicos que representa el uso de reconocidas buenas prácticas como las tecnologías limpias, la optimización de procesos, la venta de mermas, la elaboración de subproductos y la creación de fundaciones y de asociaciones civiles con fines sociales y ambientales, entre otros.

Por otro lado, también se encuentran el surgimiento de nuevas necesidades de los consumidores, quienes exigen productos y servicios amigables con el ambiente, así como la apertura de nuevos contextos competitivos. Este es el caso de los mercados verdes. Estos requieren que la empresa considere la sustentabilidad como una oportunidad y, en consecuencia, la incorporen en sus ejercicios de planeación estratégica. A pesar de esto, la normatividad en materia sustentable en México es todavía poco aplicable y escasa; en ocasiones, la corrupción permite la propagación de negocios ilegales que, de manera flagrante, incumplen las normas existentes.

Un $70 \%$ de las empresas considera que la normatividad nacional e 
internacional genera incentivos para innovar, ya que fomenta la adopción de mejores prácticas ambientales, sociales y económicas. Un 30\% de las organizaciones supone que esta normatividad establece restricciones rígidas para adoptar nuevos productos y procesos sustentables, debido a los procedimientos y trámites que conlleva.

Asimismo, un $20 \%$ de las empresas sugiere que la normatividad ambiental incide negativamente en la competitividad, por la corrupción existente y porque otros negocios la desconocen. Un $10 \%$ de las compañías plantea que esta modifica las demandas del mercado, al introducir nuevos criterios para comercializar productos y servicios.

Otro incentivo por el cual las empresas instituyen dicha gestión es la presión que ejercen los grupos de interés. Un $90 \%$ de ellas sostiene que los principales grupos que influyen en la adopción de la sustentabilidad corporativa son consumidores, proveedores, empleados y propietarios, provocados por las políticas del corporativo.

Un $40 \%$ afirma que las asociaciones en favor de la sustentabilidad son un factor influyente para la implementación de proyectos de sustentabilidad, mientras un $30 \%$ considera que son el Gobierno, las asociaciones profesionales y las políticas. El primero impone sanciones si se causa un daño al ambiente que pueda afectar la imagen y el prestigio de la empresa, así como generar demandas en contra de la organización si se perjudica el entorno. La mayoría de las empresas considera que los grupos de interés le recuerdan sus obligaciones morales para asumir un compromiso con la sustentabilidad. Algunas señalan que los stakeholders amenazan con sancionar a la compañía mediante denuncias o desprestigio, si esta no protege el hábitat.

\subsection{Motivaciones}

Un $90 \%$ de las empresas cree que la sustentabilidad representa una oportunidad de negocio, ya que le da una mejor imagen, prestigio, reconocimiento y certificaciones y fomenta la innovación, en tanto canaliza recursos en tecnologías que optimizan los procesos. Esta percepción es un factor relevante, puesto que de esta depende la aceptación o el rechazo de los proyectos.

Por otro lado, un $60 \%$ de las organizaciones sugiere que la sustentabilidad no es una opción, sino una necesidad. Sin embargo, un $10 \%$ de las empresas considera que la adopción de prácticas de sustentabilidad 
supone costos adicionales como la adquisición de tecnologías limpias o la obtención de certificaciones, entre otros. Cabe señalar que las compañías no consideran la sustentabilidad como un freno al crecimiento o como una moda, pues señalan su importancia para el desarrollo y crecimiento tanto de la organización como del país.

Con base en la percepción que demuestran las empresas hacia adoptar acciones de sustentabilidad en la estrategia de negocio, se observa que enfrentan diversos retos. Un 50\% asume que concientizar al cliente acerca de los productos o servicios amigables con el ambiente y establecer una cultura organizacional en materia sustentable son sus principales desafíos. Un $40 \%$ tiene como meta cumplir con la normatividad en tema sustentable y un $30 \%$, elaborar un sistema de medición del desempeño sustentable y establecer proyectos en esta materia, con alcances y objetivos delimitados. Además, un $10 \%$ sostiene que el reto es diseñar políticas de difusión de las acciones sustentables dentro y fuera de la empresa.

Al implementar una gestión ambiental que regule la adopción de acciones de sustentabilidad se requiere que las empresas fomenten y adquieran recursos y capacidades para ejecutarlas. Un 70\% de las empresas estima que han tenido que invertir recursos económicos para incorporar la sustentabilidad en la estrategia del negocio en aspectos como el costo de las reingenierías de procesos, con el fin de optimizar y minimizar mermas, así como en múltiples iniciativas de capacitación y sensibilización del personal.

De igual forma, un $60 \%$ de las organizaciones sostiene que ha debido adquirir tecnologías limpias para minimizar el consumo de recursos e incentivar el involucramiento de los directivos de la empresa en la adopción de proyectos de sustentabilidad. Un $10 \%$ ha requerido incentivos o beneficios gubernamentales, por medio de los programas del Estado y de las organizaciones certificadoras como el PNAA, cuya finalidad es promover la sustentabilidad. Un $60 \%$ implementa acciones de sustentabilidad en su cadena de suministro para cumplir con la normatividad ambiental. Un $40 \%$ lo hace en el seguimiento de políticas establecidas en la organización para aceptar a los proveedores y otro $40 \%$ por cumplir lineamientos que establece una industria específica para competir en el mercado.

La mitad de las empresas utiliza múltiples herramientas para incorporar la sustentabilidad en la planeación 
estratégica. No obstante, un $20 \%$ de las organizaciones no la considera en su estrategia y no utiliza herramienta alguna para identificar, diseñar e implementar mecanismos sustentables, sino que lo hacen de manera espontánea y sin que exista planeación.

\subsection{Incentivos}

El desarrollo sustentable busca un equilibrio entre lo económico, lo social y lo ambiental. En efecto, las empresas generan para sí este tipo de beneficios cuando adoptan un compromiso sustentable, sin contar su positivo efecto diferenciador de la competencia y el valor agregado que conlleva. Los beneficios económicos para las empresas son más restringidos, pero son suficientes como para no despreciarlos. Un $40 \%$ de las compañías reduce costos y abarca nuevos mercados; un $20 \%$ señala que se obtiene este tipo de beneficios al tener que innovar en productos y servicios y solo un $10 \%$ considera las posibilidades de reducir impuestos.

Por otro lado, un $70 \%$ de las compañías señala que el beneficio social está vinculado con la certificación, mientras un $60 \%$ expresa que es el prestigio y un $30 \%$ indica que son el reconocimiento de marca y los premios.
Un $60 \%$ de las organizaciones sostiene que el beneficio ambiental se relaciona con la reducción de emisiones de contaminantes; un $50 \%$, con la minimización del consumo de energía eléctrica; otro $40 \%$, con una reducción del consumo de agua y luz y un $20 \%$, con la disminución en el consumo de materiales.

En este contexto, las empresas hallan múltiples beneficios al incorporar la sustentabilidad corporativa en su estrategia de negocio. Además, se estima que es un factor que incrementa la competitividad en las organizaciones, porque los activos tangibles e intangibles son difíciles de imitar, insustituibles, escasos y valiosos. Un $86 \%$ está "totalmente de acuerdo" con que todo esto mejora la competitividad, mientras un $14 \%$ señala estar "de acuerdo" con esta consideración.

En conjunto, la mayor parte de las empresas está totalmente de acuerdo con que, al incorporar la sustentabilidad en la estrategia de negocio, se mejora la productividad y el desempeño financiero de la organización. Un $20 \%$ está "totalmente de acuerdo", otro $20 \%$ está "de acuerdo" y un $30 \%$ adoptó una posición más bien central.

$\mathrm{Al}$ asumir un compromiso con la sustentabilidad y optimizar procesos, 
en algunas empresas se ha incrementado el volumen de ventas, pues se abarcan nuevos mercados y se satisfacen nuevas necesidades de los consumidores, quienes exigen productos o servicios amigables con el ambiente. Un 30\% de las compañías está de acuerdo con que las acciones sustentables implementadas incrementan sus ventas y un $40 \%$ tiene una posición neutral.

Tras analizar los resultados de los cuestionarios y relacionarlos con la literatura, se identificaron los incentivos, las motivaciones y los benefi- cios que tienen las empresas cuando instituyen una gestión ambiental.

\subsection{Incentivos, motivaciones y beneficios de una gestión ambiental}

Los incentivos para las empresas se relacionan con el corporativismo verde, al dar cumplimiento a la normatividad ambiental establecida por los Gobiernos y seguir un lineamiento estratégico desarrollado por el corporativo, que exigen el cumplimiento de políticas en favor de la sustentabilidad (figura 1).

\section{Figura 1. Incentivos para implementar una gestión ambiental}
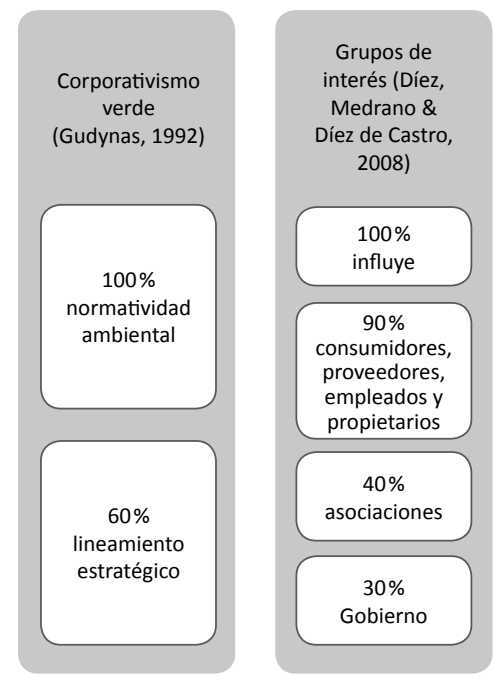

Fuente: elaboración propia.

Otro incentivo es la presión que ejercen los grupos de interés para que las organizaciones consideren

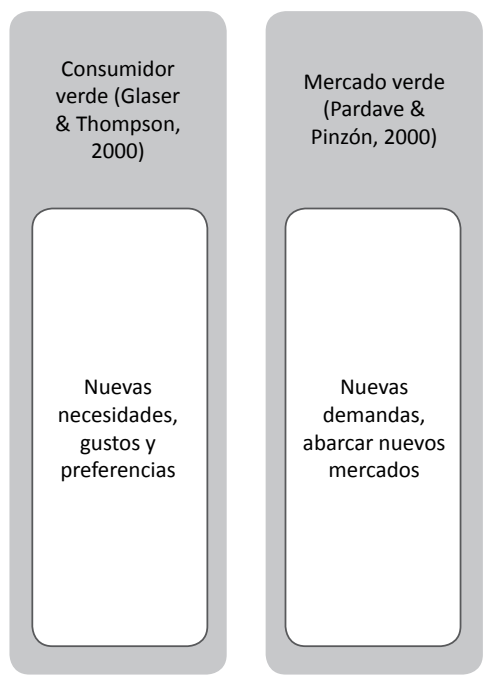

la sustentabilidad en la estrategia de negocio, los cuales influyen de manera sistemática para que estas 
adopten un comportamiento sustentable. Entre los stakeholders más destacados se encuentran los consumidores, los proveedores, los empleados, los propietarios, las asociaciones civiles o no gubernamentales y el Gobierno.

El consumidor verde demanda productos y servicios amigables con el ambiente, debido a que sus necesidades han ido cambiando. Para investigadores como Glaser y Thompson (2000), estos cambios tienen que ver con su forma de vida y su comportamiento adquisitivo, en tanto para Tannery Wölfing Kast (2003) se relacionan con actitudes, conocimiento, comportamiento, barreras morales y contextuales, características socioeconómicas, condiciones de vida y el tipo de tiendas propio de la organización. Estos aspectos determinan el comportamiento del consumidor, quien influirá en los productos y servicios que ofrece la empresa.

Además, con el cambio de las necesidades de los consumidores y la incorporación de la sustentabilidad en el proceso de elaboración de productos y servicios, ha surgido un nuevo mercado. Esto se corrobora en los escenarios internacionales de intercambio, los cuales exigen cumplir con una normativa ambiental como requisito para competir y mantenerse. En los mercados nacionales tam- bién se procura generar valor agregado y satisfacer los requerimientos de los consumidores.

Maldonado, Rivas, Molina y Flores (2007) indican que, en efecto, el mercado ha cambiado y que la necesidad de incorporar la sustentabilidad en el proceso de la elaboración de productos y servicios se ha acentuado, porque constituye un requisito para competir en los mercados internacionales.

Como se muestra en la figura 2, las motivaciones de las empresas para implementar una gestión ambiental son cuatro, en particular. La primera es el perfil empresarial. Las compañías consideran que la sustentabilidad representa una oportunidad de negocio, ya que esta les da una mejor imagen, prestigio, reconocimiento y certificaciones y fomenta la innovación, aunque deban invertir en tecnologías que optimicen los procesos. Esta percepción es un factor relevante, porque de esta depende la aceptación o el rechazo de los proyectos.

Las organizaciones asumen que la sustentabilidad no es una opción, sino una necesidad. La sociedad demanda que las empresas adopten una actitud proactiva en el marco de la economía verde. Se estima que es responsabilidad de la compañía asumir 
su rol en la relación tripartita empresa-sociedad-Gobierno y es conside- rado un tema sustancial (Bowen, 2000; Hart \& Milstein, 2003).

Figura 2. Motivaciones para implementar una gestión ambiental

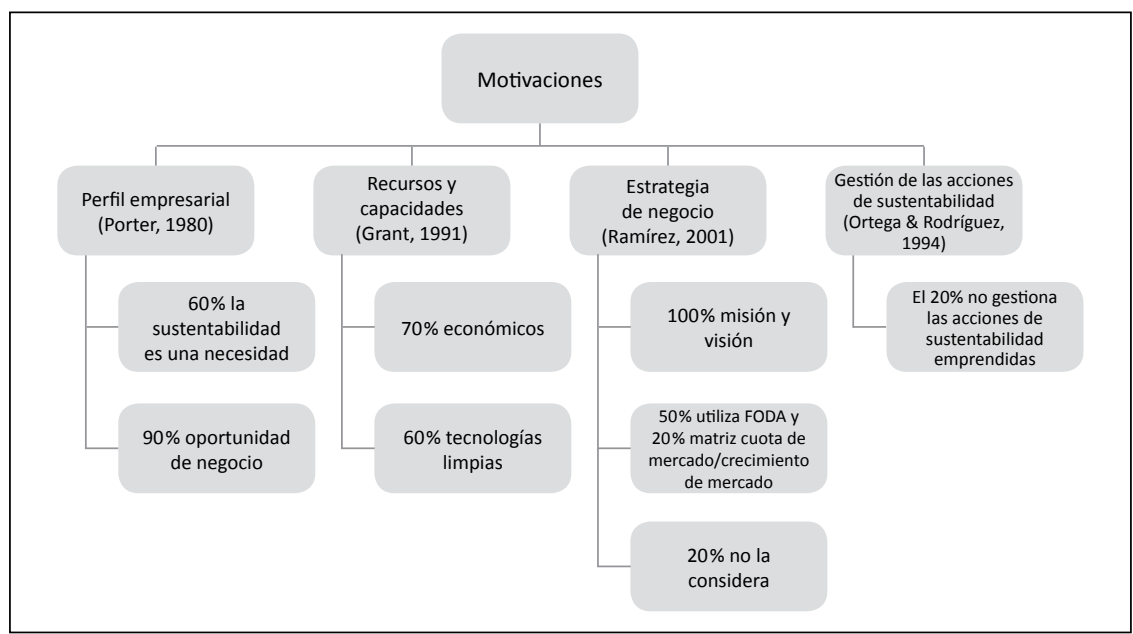

Fuente: elaboración propia.

La segunda motivación esencial que tienen las empresas son los recursos y las capacidades vinculados con la existencia de una gestión ambiental que regule la adopción de acciones en materia de sustentabilidad —el cuarto tipo de motivación básica(figura 2). Se trata de recursos como los económicos que han canalizado las organizaciones para incorporar la sustentabilidad en su estrategia de negocio. Un ejemplo de ello es el costo de las reingenierías de procesos con el fin de optimizarlos, minimizar mermas, capacitar y sensibilizar al personal. Las empresas, además, tienen que adquirir tecnologías limpias, para reducir el consumo de re- cursos y se ven enfrentadas a tratar de lograr un alto involucramiento por parte de los directivos de la empresa en la adopción de proyectos de sustentabilidad (De Burgos \& Céspedes, 2001).

En la estrategia de negocio, la sustentabilidad se evidencia en la misión y la visión, que comunican a todos los miembros de la organización el compromiso empresarial con el desarrollo sustentable - el tercer tipo de motivación básica- (figura 2). Sin embargo, solo algunas empresas consideran este aspecto en el análisis de fortalezas, oportunidades debilidades y amenazas (FODA) o en 
el realizado con la matriz cuota de mercado/crecimiento de mercado. Otras compañías ni siquiera lo estiman como parte de su estrategia de negocio y, en consecuencia, no ejecutan sus acciones de sustentabilidad corporativa.

Como se muestra en la figura 3 , los beneficios que obtienen las empresas por implementar una gestión ambiental también son económicos. En efecto, reducen sus costos, al optimizar los procesos productivos e incursionar en nuevos mercados, en atención a las necesidades cambiantes de los consumidores.
Los beneficios sociales también son visibles. En particular, se presentan al adquirir certificaciones en materia sustentable, lo que brinda a las organizaciones la oportunidad de competir en mercados internacionales, de incrementar su prestigio, su reconocimiento de marca $y$, entre otras cosas, obtener premios.

Los beneficios ambientales, por su parte, son la reducción de emisiones, el consumo de energía eléctrica, agua y luz y materiales, así como una mayor competitividad y productividad y un mejor desempeño financiero mediante el incremento de las ventas.

Figura 3. Beneficios de implementar una gestión ambiental

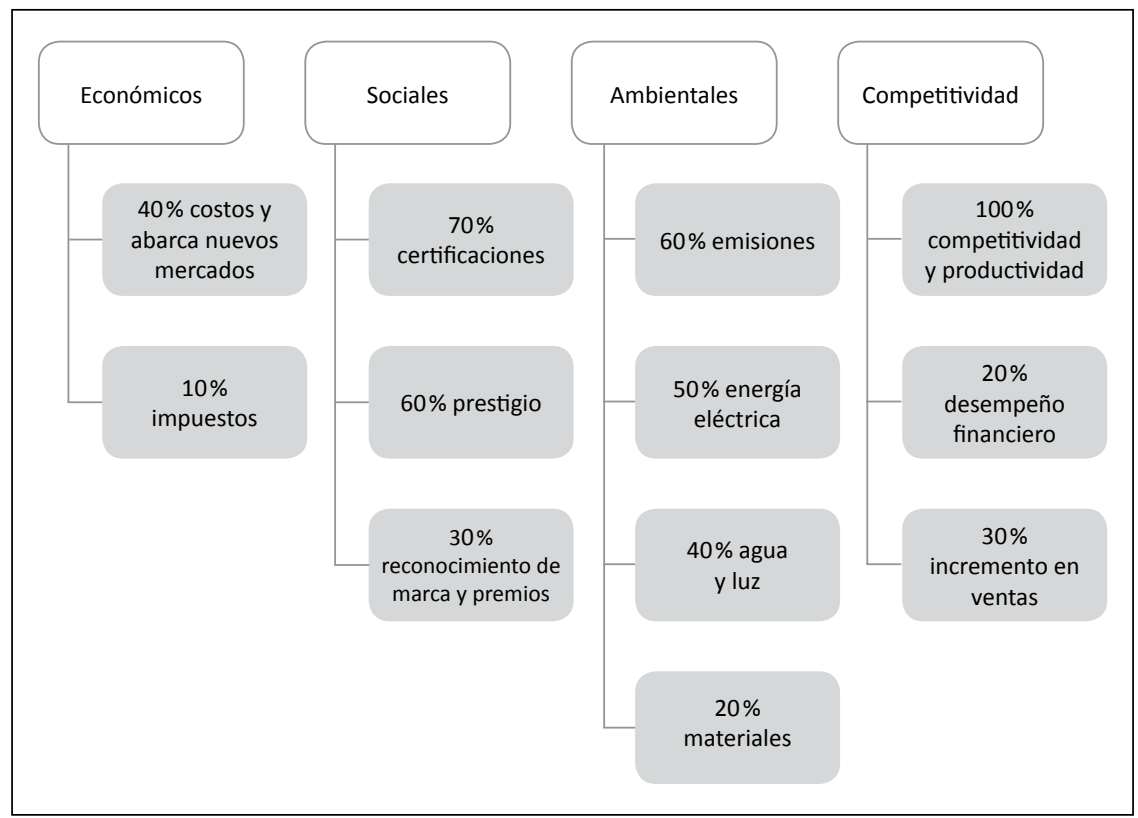

Fuente: elaboración propia. 
A pesar de lo encontrado, algunas empresas no contemplan la gestión de las acciones de sustentabilidad en la planeación estratégica. Esto hace que el diseño, la implementación y el control de estas actividades sean más bien improvisados y espontáneos. Las empresas deben considerar la sustentabilidad en la planeación estratégica, con el fin de que pueda ser adoptada en la estrategia de negocio, en el marco de la economía verde. El desarrollo sustentable, como se ha indicado, no es una moda, sino una necesidad. Para abordar este tema, la empresa, la sociedad y el Gobierno deben asumir su responsabilidad individual y las organizaciones deben verlo como una verdadera oportunidad de negocio.

\section{CONCLUSIONES}

La escasez de recursos, el calentamiento global y la disminución de la calidad de vida de la sociedad son algunos de los factores por los cuales las empresas deben asumir su responsabilidad en la relación empresa-sociedad-Gobierno, para cumplir con la premisa del desarrollo sustentable. Como resultado, los grupos de interés dirigen su atención a la generación de soluciones sustentables y presionan a la empresa para que integre la sustentabilidad en su estrategia de negocio. Sin embargo, la mayoría de ellas desconoce la existencia de incentivos, motivaciones y beneficios de implementar una herramienta que regule las acciones de sustentabilidad corporativa (Rodríguez, Villareal, Giachetti \& Banchero, 2002).

En el caso de México, las responsabilidades en materia sustentable son poco aplicables y algunas empresas asumen un compromiso de respeto, conservación y protección del ambiente por medio de la certificación de Industria limpia. En este contexto, el nexo entre la sustentabilidad, las condiciones de la industria, la empresa y la competencia se ve marcado por los aportes de varios autores expertos en el tema. Ellos consideran que los incentivos, las motivaciones y los beneficios son una pauta para que las compañías instituyan la sustentabilidad en la estrategia de negocio mediante de la gestión ambiental.

Se identificó que la gestión ambiental tiene como finalidad planear, organizar, dirigir y controlar las acciones de sustentabilidad que defina la empresa, cuyo incentivo es el corporativismo verde que, aunado a la idea de cumplir con la escasa y poco aplicable normatividad ambiental, en México guía a múltiples organizaciones.

Los grupos de interés, como los consumidores, proveedores, empleados 
y propietarios, exigen la incorporación de prácticas de sustentabilidad mediante la aportación de nuevas ideas y recuerdan a los actores sus obligaciones morales con la sociedad, a pesar de la existencia de barreras del sector que impiden a las empresas adoptar un compromiso sustentable. Entre estas se encuentran aspectos como los costos que representa lograr una certificación, adquirir tecnologías, la reingeniería de procesos y la corrupción, entre otros.

Las motivaciones de las empresas para implementar esta gestión son: 1) su perfil empresarial, que muestra la necesidad de considerar la sustentabilidad en la estrategia de negocio; 2) el hecho de que pueda representar una oportunidad de negocio, en el que los recursos y las capacidades a obtener o fomentar son la adquisición de tecnologías limpias, el desarrollo de cursos de capacitación, la sensibilización del personal y la innovación en productos y procesos, entre otros; 3) el desarrollo de acciones de manera eficiente y eficaz que contribuyen a la rentabilidad, y 4) la mejora de la posición competitiva de la organización en el mercado.

Los beneficios para las empresas que implementan una gestión ambiental son: minimización de costos, al utilizar tecnologías limpias; obtención de mayores ingresos; apertura a nue- vos mercados; incremento del prestigio de la firma; reconocimientos, premios y certificaciones; reducción del consumo de materiales, agua, energía y luz, entre otros. Así, con el cumplimiento de la premisa de desarrollo sustentable se consiguen beneficios económicos, sociales y ambientales. Esto genera un efecto diferenciador de la competencia, pues aumenta su productividad, desempeño financiero y comercial, prestigio e imagen y contribuye, incluso, a alcanzar un incremento en las ventas.

\section{REFERENCIAS}

Auld, G. (2014). Constructing private governance: The rise and evolution of forest, coffee, and fisheries certification. New Haven: Yale University Press.

Ayuntamiento de Socuéllamos. (2000). Guía para el desarrollo sostenible de la pequeña ymediana empresa. Socuéllamos: Autor.

Banerjee, S. B. (2002). Corporate environmentalism: The construct and its measurement. Journal of Business Research, 55(3), 177-191.

Barba, A. A. \& Solís, P. C. (2000). La empresa sustentable en una sociedad de riesgo. Iztapalapa (48), 163-182.

Barrow, C. J. (2006). Environmental management for sustainable development (2a ed.). Londres: Routledge. 
Belcham, A. (2015). Manual of environmental management. Londres: Routledge.

Binder, M., Jänicke, M. \& Petschow, U. (eds.). (2001). Green industrial restructuring: International case studies and theoretical interpretations. Berlin: Springer.

Black, T. R. (2009). Doing quantitative research in the social sciences: An integrated approach to research design, measurement and statistics. Londres: SAGE.

Bowen, F. E. (2000). Environmental visibility: A trigger of green organizational response? Business Strategy and the Environment, 9(2), 92-107.

Cajiga, C. J. (2012). El concepto de responsabilidad social empresarial. México D. F.: Centro Mexicano para la Filantropía.

Chamorro, A. (2001). El marketing ecológico. 5campus.org, Medio Ambiente. Recuperado de http:// ciberconta.unizar.es/Leccion/ ecomarketing/ecomarketing.pdf

Cleveland, C. J., Stern, D. I. \& Costanza, R. (eds.). (2001). The economics of nature and the nature of economics. Northampton: Edward Elgar.

Daly, H. E. (1996). Beyond growth: the economics of sustainable development. Boston: Beacon Press.

De Burgos, J. \& Céspedes, J. (2001). Environmental performance as an operations objective. Inter- national Journal of Operations \& Production Management, 21(12), 1553-1572.

Edwards, A. J. (2001). ISO 14001 Environmental certification step by step. Oxford: ButterworthHeinemann.

Elliott, J. A. (2006). An introduction to sustainable development (3a ed.). Nueva York: Routledge, Taylor \& Francis Group.

Enders, J. C. \& Remig, M. (eds.). (2015). Theories of sustainable development. Londres: Routledge, Taylor \& Francis Group.

Glaser, L. \& Thompson, G. (2000). Demand for organic and conventional beverage milk. Recuperado de http://faculty.ksu. edu.sa/62311/Research\%20Library/19.pdf

Gudynas, E. (1992). Los múltiples verdes del ambientalismo latinoamericano. Nueva Sociedad, (122), 104-115.

Gulbrandsen, L. H. (2010). Transnational environmental governance: The emergence and effects of the certification of forests and fisheries. Cheltenham: Edward Elgar.

Hart, S. \& Milstein, M. (2003). Creating sustainable value. $A c a-$ demy of management executive, 17(2), 56-69.

Hernández, R., Fernández, C. \& Baptista, P. (2009). Metodología de la investigación. México: McGraw-Hill. 
Jacobsen, J. (2011). Sustainable business and industry: Designing and operating for social and environmental responsibility. Milwaukee: ASQ Quality Press.

Lacruz, F. F. (2005). La empresa ambientalmente responsable. Una visión de futuro. Economía (21), 39-58.

Lawler III, E. E., Worley, C. G. \& Creelman, D. (2011). Management reset: Organizing for sustainable effectiveness. San Francisco: Jossey-Bass.

López, M., Molina, J. \& Claver, E. (2008). Análisis de los factores que condicionan la percepción del directivo sobre el medio ambiente. Un estudio Qual/Quan. Cuadernos de Economía y Dirección de la Empresa (37), 123-172.

Maldonado, B., Rivas L. A., Molina, D. \& Flores, J. L. (2007). Análisis de los modelos de marketing ambiental. Universidad \& Empresa, 9(12), 20-38.

Menon, A. \& Menon, A. (1997). Environpreneurial marketing strategy: the emergence of corporate environmentalism as market strategy. Journal of Marketing, (61), 51-67.

Pacheco, S. V., Pavón, S. T. \& Mejía, P. G. (2002). Prospectiva de la gestión ambiental en México. Recuperado de http://www. bvsde.paho.org/bvsaidis/mexico26/viii-039.pdf
Procuraduría Federal de Protección al Ambiente [Profepa]. (2014). Programa Nacional de Auditoría Ambiental Profepa. Recuperado de http://www.profepa. gob.mx/innovaportal/v/26/1/ $\mathrm{mx} /$ programa_nacional_de_auditoria ambiental.html

Rainey, D. L. (2006). Sustainable business development: Inventing the future through strategy, innovation, and leadership. Cambridge: Cambridge University Press.

Ramírez, T. A., Sánchez, N. J. \& García, C. A. (2004). El desarrollo sustentable: interpretación y análisis. Revista del Centro de Investigación. Universidad La Salle, 6(21), 55-59.

Ritchie, J. (2013). Qualitative research practice: A guide for social science students and researchers ( $2 a$ ed.). Thousand Oaks: SAGE.

Rodríguez, M. A., Villareal, M., Giachetti, M. I. \& Banchero, M. E. (2002). Costos ambientales, su impacto en las empresas. Recuperado de http://www.econ. uba.ar/www/seminario/g319/ Costos \%20Ambientales, $\% 20$ $\mathrm{Su} \% 20$ impacto\%20en $\% 20$ las\%20empresas.doc

Rogers, P. P., Jalal, K. F. \& Boyd, J. A. (2008). An introduction to sustainable development. Londres: Earthscan. 
Rushton, K. (2002). Business ethics: A sustainable approach. Business Ethics: A European Review, 11(2), 137-139.

Sachs, J. (2015). The age of sustainable development. Nueva York: Columbia University Press.

Secretaría del Medio Ambiente y Recursos Naturales [Semarnat]. (2014). Compendio de estadisticas ambientales. Edición 2014. Recuperado de http://apps1.semarnat.gob.mx/dgeia/compendio_2014/dgeiawf.semarnat. gob.mx_8080/ibi_apps/WFServlet $772 \overline{6} . \mathrm{html}$

Soubbotina, T.P.(2004). Beyondeconomic growth: An introduction to sustainable development ( $2 a$ ed.). Washington: World Bank.

Tanner, C. \& Wölfing Kast, S. (2003). Promoting sustainable consumption: Determinants of green purchases by swiss consumers. Psychology \& Marketing, 20(10), 883-902.

Teddlie, C. \& Tashakkori, A. (2009). Foundations of mixed methods research: Integrating quantitative and qualitative approaches in the social and behavioral sciences. Thousand Oaks: SAGE.
Wayne, A. L. (2000). ISO 14000: un sistema de administración ambiental con oportunidades para contadores y otros consultores. Contaduría y Administración, (198), 55-66.

Wheelen, T. L., Hunger, J. D., Hoffman, A. N. \& Bamford, C. E. (2014). Strategic management and business policy: Globalization, innovation, and sustainability (14a ed.). Boston: Pearson.

Wild, J. J. \& Wild, K. L. (2014). International business: The challenges of globalization (7a ed.). Boston: Pearson.

Williams, O. F. (2014a). Sustainable development: The un millennium development goals, the un global compact, and the common good. Notre Dame: University of Notre Dame Press.

Williams, O. F. (2014b). Corporate social responsibility: The role of business in sustainable development. Londres: Routledge, Taylor \& Francis Group.

World Commission on Environment and Development [WCED]. (1987). Our common future. Oslo: United Nations. 